\title{
CONSONANCIA - DISONANCIA ENTRE LAS VALORACIONES DE LOS DOCENTES Y SU APLICACIÓN SEGÚN LA PERCEPCIÓN DE LOS ESTUDIANTES
}

\author{
Dr. Manuel Miljanovich Castilla ${ }^{1}$
}

\begin{abstract}
Al comparar las valoraciones que una muestra de profesores sanmarquinos formula sobre aspectos sustanciales de la institución universitaria con la percepción que tienen los estudiantes de su aplicación en la praxis docente, se constata un alto grado de consonancia en torno a los valores relacionados con la democracia ( respeto a la persona humana, cultivo del diálogo, búsqueda de la verdad en la investigación científica y humanística) y una apreciable disonancia respecto a los valores político sociales, de proyección social, pedagógicos y ético - morales. No hay variaciones según las carreras que siguen los estudiantes. El grado de disonancia en los primeros años de estudios se incrementa en los últimos años, con algunas variaciones según las áreas de valores y las carreras.
\end{abstract}

When comparing the valuations from a sample that San Marcos' professors formulate about the university's substantial aspects with the students' perception of its application in educational practice, a high consonance degree is verified around the values related with democracy ( the respect to the human person, the cultivate of dialogue, the search of the truth in the scientific and humanistic investigation ) and an appreciable dissonance regarding the political and social values of social, pedagogic and ethical moral, of social, pedagogic and the ethical- moral projection. There are no variations according the careers that the students selected. The dissonance degree in the first years of studies is increasing in the last years, with some variations according tú the values' fields and careers.

PALABRAS CLAVE: Comparación valoraciones teóricas docentes percepción aplicación estudiantes

KEY WORDS: Comparison valuations theoretical educational perception application students.

\footnotetext{
${ }^{1}$ Participaron en la Investigación: Psic. María Clotilde Atalaya Pisco, Mg. Rosa Elena Huerta Rosales
} 


\section{INTRODUCCIÓN}

El escenario nacional del Perú de hoy presenta como una de sus características más marcadas una compleja y arraigada crisis moral, manifestada en diversas formas de corrupción, las mismas que se concretizan en percepciones, actitudes y comportamientos antiéticos individuales y grupales.

Hay consenso en la opinión pública acerca de la existencia de dicha crisis y de la urgencia de emprender acciones sistemáticas de gran envergadura, de mediano y largo plazo, para revertir tal situación. Sin embargo, la concepción y planeamiento de dichas acciones no pueden basarse en impresiones generales sino en los resultados de investigaciones científicas que contribuyan con información válida y confiable a un diagnóstico certero de los referidos males sociales.

En el marco conceptual esbozado se inscribe el problema a investigar del presente estudio y cuya formulación es la siguiente: ¿Cuál es el grado de consonancia-disonancia entre el nivel de importancia que los profesores asignan a algunos 2 spectos y actividades substanciales de la institución universitaria y la percepción que tienen los estudiantes de la aplicación de tal valoración en la práctica docente?

Las variables sustantivas involucradas en el problema en cuestión son: a) Las valoraciones de los docentes y b) la percepción que tienen los estudiantes de la aplicación de tales valoraciones en la práctica docente. Las variables controladas son: a) Las Escuelas Académico-Profesionales o carreras a las que pertenecen los estudiantes y en las que enseñan los profesores y b) el tiempo de permanencia de los estudiantes en la universidad.

Sobre la base de las observaciones que realizan cotidianamente numerosos docentes de diversas especialidades, preocupados por la problemática de San Marcos y del país, de las que tomamos conocimiento a través de la interacción propia de la vida académica o de conversatorios algo más formales, planteamos la hipótesis de que en San Marcos y en otras universidades existe un grado apreciable de disonancia entre el grado de importancia que los docentes asignan a algunos aspectos y actividades substancial es de la universidad y la percepción que tienen los estudiantes de la aplicación de tales valoraciones en la práctica docente.

El objetivo general, como en toda investigación, fue poner a prueba nuestra hipótesis central y los objetivos específicos estuvieron referidos a examinar las combinaciones de las categorías de las variables sustantivas con las categorías de las variables controladas, así como analizar la especificidad de determinadas clases de respuestas en atención a su particular significado desde nuestra perspectiva.

El estudio se llevó a cabo en el ámbito de la UNMSM, durante el año académico 2002 y parte del 2003. Se tiene previsto replicarlo, con los consiguientes ajustes, en otras universidades.

La justificación de la investigación realizada es evidente si, por un lado, tenemos en cuenta la crisis de valores y el incremento de la corrupción producidos en el país en los últimos lustros y si, por otro lado, se considera que la hipótesis planteada comporta la posibilidad de un problema ético de inautenticidad que tendría la perniciosa propiedad de impactar en los estudiantes, como un modelo social de comprensión y de comportamiento.

En ese sentido y teniendo en cuenta el efecto multiplicador que el modelo ético docente suele ejercer en los estudiantes, es de esperarse que dicho modelo tenderá a reproducirse en el ulterior desempeño profesional de éstos. Por lo tanto, juzgamos de la mayor importancia descartar o, por el contrario, detectar e identificar las inconsistencias y contradicciones entre los valores que se proclaman y las conductas y hechos reales, a fin de plantear con sólidos argumentos la urgencia de realizar acciones correctivas específicas en el citado campo. 


\section{MÉTODO}

Teniendo como universo la UNMSM, se diseñó una muestra representativa de docentes y estudiantes, pertenecientes a la totalidad de Escuelas Académico Profesionales. Sin embargo, las dificultades surgidas en la aplicación de los instrumentos determinaron que, en la práctica, los subconjuntos de docentes y estudiantes se redujeran a la condición de muestras intencional es, con una magnitud de 150 docentes y 400 estudiantes, pertenecientes a 9 carreras.

Los instrumentos de medición y obtención de la información fueron una encuesta de opinión para los docentes y una escala de percepción para los estudiantes, con la particularidad de que ambos tuvieron los mismos reactivos, pero diferentes instrucciones y alternativas de respuesta. Los reactivos, en número de 20, consistieron en enunciados referentes a diversos aspectos de la institución universitaria y a las actividades que les son propias.

Las alternativas para los docentes fueron:

Imprescindible, Importante, Poco importante y Carente de importancia. Con ellas se les pedía que calificaran el contenido de los enunciados. Las alternativas para los estudiantes fueron: Siempre, Con frecuencia, Casi nunca y Nunca, con las cuales se les pedía que indicaran la frecuencia con la que, según la experiencia de cada quien, ocurría lo referido por los enunciados.

Los instrumentos fueron construidos mediante un trabajo en equipo y a través de un proceso sistemático que concluyó con los reajustes consecuentes a un pretest de apreciable envergadura.

En atención al nivel ordinal de las variables en el diseño ya las necesidades específicas de procesamiento de la información, se emplearon los porcentajes, su error estándar y su intervalo de confianza, así como el estadístico CHI cuadrado (CHP) de una entrada y de dos entradas, en cuadros de contingencia. 


\section{RESULTADOS}

Los ítemes componentes de los instrumentos de medición fueron clasificados, para efectos del procesamiento de la información, en cinco arcas de valores, en la forma siguiente:

\section{Áreas de valores e ítemes}

\section{VALORES ETICO - MORALES}

1.- Búsqueda de la verdad en la investigación científica y humanística

3.- Honestidad en las actividades académico-administrativas

12.- Consistencia entre las declaraciones y los actos

16.- Autenticidad en las opciones ideológicas asumidas

-II. VALORES RELACIONADOS CON LA DEMOCRACIA

2.- Respeto por la libertad de pensamiento y de expresión

4.- Respeto a la persona humana

7.- Disposición para el diálogo

8.- Tolerancia en el diálogo

\section{- IV. VALORES POLÍTICO-SOCIALES}

10.- Análisis y reflexión sobre la problemática del país

11. Compromiso con la recuperación moral del país.

14.- Compromiso con el desarrollo del país

15.- Examen crítico de las políticas gubernamentales sobre los problemas económico- sociales del país.

19.- Compromiso con la afirmación de la identidad nacional.

20.- Rechazo a los prejuicios étnicos y regionales.

\section{- V. VALORES RELACIONADOS CON LA PROYECCION SOCIAL}

17.- Proyección social universitaria hacia la comunidad.

18.- Solidaridad con los sectores poblacionales más desfavorecidos. 
Cuadro $\mathrm{N}^{\circ} 1$ : Respuestas de docentes y estudiantes a las cuestiones valorativas planteadas por los instrumentos de obtención de la información, según sus respectivas opciones y según áreas de agrupación de los Ítemes

\begin{tabular}{|c|c|c|c|c|c|c|c|c|}
\hline \multicolumn{4}{|c|}{ DOCENTES } & \multirow[b]{2}{*}{ TREMS } & \multicolumn{4}{|c|}{ ESTUDIANTES } \\
\hline $\begin{array}{c}\text { IMPRE } \\
\text { SCIND } \\
\text { I } \\
\text { BLE }\end{array}$ & $\begin{array}{l}\text { IMPOR } \\
\text { TANTE: }\end{array}$ & $\begin{array}{l}\text { POOD } \\
\text { IMPO } \\
\text { RTAN } \\
\text { TIE }\end{array}$ & $\begin{array}{l}\text { CAREN } \\
\text { TE DE } \\
\text { IMPOR } \\
\text { TAKCI }\end{array}$ & & $\begin{array}{l}\text { SIEM- } \\
\text { PRE }\end{array}$ & $\begin{array}{c}\text { CON } \\
\text { FREC } \\
\text { UENCI } \\
\text { A }\end{array}$ & $\begin{array}{c}\text { CASI } \\
\text { FUN- } \\
\text { CA }\end{array}$ & $\begin{array}{c}\mathrm{NU} \\
\mathrm{NC} \\
\mathrm{A}\end{array}$ \\
\hline$\%$ & $\%$ & $\%$ & $\%$ & I VALORESETICO-MORALES & $\%$ & $\%$ & $\%$ & $\%$ \\
\hline 6884 & 30.4 & 0.72 & - & $\begin{array}{l}\text { 1. Búsqueda de a verdad en la investigación } \\
\text { cientific y humanistica }\end{array}$ & 23.00 & 55.56 & 20.67 & 0,77 \\
\hline 83.45 & 1655 & $m$ & - & $\begin{array}{l}\text { 3. Horestidad en las actividades academico- } \\
\text { administrativas }\end{array}$ & 2161 & 4427 & 2891 & 5.21 \\
\hline $51: 80$ & 4401 & 3.47 & 0.72 & 12- Consistencia entre las dechraciones y be actos & 1398 & 4565 & 37.20 & 3.17 \\
\hline \multirow[t]{2}{*}{27.66} & 4894 & 19.15 & 4.25 & 16- Autenticidad en las opciones ideológices acumidas & 12.21 & 47.27 & 36.62 & 390 \\
\hline & & & & $\begin{array}{l}\text {-II VALORES RELACIONADOS CON LA } \\
\text { DEMOCRACIA }\end{array}$ & & & & \\
\hline 70.75 & 2925 & -. & $\cdots$ & $\begin{array}{l}\text { 2. Respeto por ha liberted de pereamiento y de } \\
\text { expresion }\end{array}$ & 42.16 & 4499 & 11.82 & 1.03 \\
\hline 80.85 & 18.44 & 0.71 & $\cdots$ & 4. Respeto a la persona hunana & 43.33 & 45.13 & 10.26 & 1.28 \\
\hline 41.78 & 52.05 & 5.48 & 0.69 & 7. Dispocición para el diábgo & 2788 & 49.62 & 2199 & 0.51 \\
\hline \multirow[t]{2}{*}{36.17} & 5816 & 4.25 & 1.42 & 8. Tolerancia en el diflogo & 2098 & 55.18 & 22.80 & 104 \\
\hline & & & & - III VALORES PEDAGOCICOS & & & & \\
\hline 55.72 & 42.14 & 2.14 & - & 5. Actulizacion del proceso ensefranza - aprendizaje & 13.11 & 52.44 & 31.88 & 2.57 \\
\hline 35.97 & 60.43 & 3.60 & $-\cdots$ & 6. Objetividad en la calificación del aprendiraje & 15.17 & 5990 & 2339 & 1.54 \\
\hline 58.27 & 35.97 & 432 & 1.44 & 9. Compromiso con la calidad de la educación & 17.65 & 50.13 & 28.90 & 3.32 \\
\hline \multirow[t]{2}{*}{3213} & 5355 & 432 & -. & 13- Cumplimento de b progamado on el syllibus & 970 & $59 \mathrm{~m}$ & $27 n$ & 387 \\
\hline & & & & IV. VALORES POLTHCO - SOCIALES & & & & \\
\hline 30.50 & 62.41 & 4.26 & 284 & 10. Anélisis y reflexión sobre ha problemática del pais & 20.81 & 46.95 & 28.93 & 3.30 \\
\hline 48.25 & 44.75 & 490 & 2.10 & 11. Compromiso con la recuperación moral del pais. & 18.16 & 40.15 & 36.06 & 5.63 \\
\hline 36.23 & 5580 & 652 & 1.45 & 14. Cotupromiso con el desarmolb del pais & 15.18 & 45.55 & 35.08 & 4.19 \\
\hline 2302 & 59.00 & 15.83 & 2.15 & $\begin{array}{l}\text { 15. Exaron entico de las politicas gubemamentales } \\
\text { sobre los problemas económicos y sociales del }\end{array}$ & 11.28 & 4179 & 39.74 & 7,18 \\
\hline 36.17 & 4823 & 12.76 & 284 & $\begin{array}{l}\text { 19. Compromiso con la afirmación de la identidad } \\
\text { ancional. }\end{array}$ & 19.90 & 46.43 & 2985 & 3.82 \\
\hline \multirow[t]{2}{*}{37.50} & 45.14 & 1250 & 446 & 20- Rechazo a bo prejuicios 6tricos y regionales. & 25.38 & 43.59 & 2436 & 6.67 \\
\hline & & & & $\begin{array}{l}\text { V. VALORES RELACIONADOS CONLA } \\
\text { PROYECCION SOCLAL }\end{array}$ & & & & \\
\hline 31.88 & 58.70 & 725 & 2.17 & $\begin{array}{l}\text { 17. Proyectión Sociel Unversitena hacia la } \\
\text { comunidad }\end{array}$ & 1330 & 42.71 & 38.81 & 5.12 \\
\hline 2993 & 5839 & 730 & 438 & $\begin{array}{l}\text { 18. Soldiandad con las sectores poblacionales más } \\
\text { desfavorecidios }\end{array}$ & 16.37 & 3804 & 38.79 & 6.80 \\
\hline
\end{tabular}

El cuadro $\mathrm{N}^{\circ} 1$ presenta las respuestas de docentes y estudiantes a cada uno de los ítemes, en términos de porcentajes y por áreas de valores. Se observa, prima facie que, como corresponde a la cultura compartida entre docentes y estudiantes, hay en los diferentes ítemes predominio cuantitativo de las consonancias sobre las disonancias entre las valoraciones de los docentes y la percepción de los estudiantes. Sin embargo, el análisis de los datos pone también de relieve un variado conjunto de disonancias que, aunque son 
cuantitativamente menores que las consonancias poseen un significado digno de tenerse en cuenta, por cuanto revelan una problemática que concierne a aspectos sustanciales de la formación (no sólo instrucción) de profesionales, investigadores y futuros dirigentes del país.

En lo que sigue exponemos y comentamos las más categóricas consonancias y las principales disonancias, por áreas de valores, sin hacer distinciones respecto a las variables controladas, basándonos para ello en los cuadros $\mathrm{N}^{\circ} 1$ y $\mathrm{N}^{\circ} 2$.

El cuadro $\mathrm{N}^{\circ} 2$ tiene el mismo contenido que el cuadro $\mathrm{N}^{\circ} 1$, con la diferencia de que en aquél las alternativas de respuesta, tanto de docentes como de estudiantes, se han reducido de cuatro a dos para facilitar la comprensión del conjunto. Se han fusionado $1 \mathrm{~m}$. prescindible e Importante, así como Poco Importante y Carente de Importancia en lo que toca a docentes. Se ha hecho lo mismo con Siempre y Con Frecuencia, así como con Casi Nunca y Nunca, en lo que concierne a estudiantes.

En cuanto a consonancias categóricas, se advierte que en los ítemes 2 (Respeto por la libertad de pensamiento y de expresión) y 4 (Respeto a la persona humana), ambos pertenecientes al área de Valores relacionados con la democracia, la valoración de los docentes es muy alta: $100 \%$ (sumando 70.75 y 29.25: Imprescindible e Importante) y 99.29\% (sumando 80.85 y 18.44: Imprescindible e Importante). Frente a los mismos ítemes, el $87.15 \%$ de los estudiantes (sumando 42.16 y 44.99: Siempre y Con frecuencia, respectivamente) y el $88.46 \%$ (sumando 43.33 y 45.13 ) declaran que Siempre o Con frecuencia, tales valoraciones se plasman en la realidad. Sólo el 12.85\% (sumando 10.26 y 1.28 ) y el $11.54 \%$ (sumando $10.26 \mathrm{Y} 1.28 \%$ ) consideran que Casi nunca y Nunca se cumplen en la realidad.

En el área de los valores Ético-morales, se advierte que las mayores disonancias entre las valoraciones de los docentes y la percepción de los estudiantes se encuentran en los dos últimos ítemes, 12: (Consistencia entre las declaraciones y los actos) y 16: (Autenticidad en las opciones ideológicas asumidas). El indicador más elocuente de ello es la elevada importancia que conceden los docentes a las cuestiones referidas por los citados ítemes 12 y 16, a saber, en el ítem $12: 95.81 \%$, que es resultado de sumar e Imprescindible (51.80\%) e Importante $(44.01 \%)$; y en el ítem $16: 76.60 \%$ que resulta de sumar Imprescindible $(27.66 \%)$ e Importante ( $48.94 \%)$.

Frente a tales valoraciones, los estudiantes manifiestan una percepción desfavorable en un apreciable porcentaje. Así, en el ítem 12: el $40.37 \%$, que resulta de sumar Casi nunca (37.20\%) y Nunca (3.17\%); y en el ítem 16: el $40.52 \%$, que es el resultado de sumar Casi nunca (36.62 \%) y Nunca (3.90\%). Algo similar ocurre con el ítem 5: (Actualización del proceso de enseñanzaaprendizaje (Valores pedagógicos): mientras que los docentes le asignan una gran importancia (Imprescindible: 55.72 e Importante: 42.14, y sumadas ambas: 97.86\%), los estudiantes, con un porcentaje de $34.45 \%$ (sumando 31.88 y 2.57) consideran que Casi nunca o Nunca tal valoración se plasma en la realidad. 
Cuadro $\mathrm{N}^{\circ}$ 2: Respuestas de docentes y estudiantes a las cuestiones valorativas planteadas por los instrumentos de obtención de la información, según opciones reducidas de cuatro a dos y según áreas de agrupación de los ítemes.

\begin{tabular}{|c|c|c|c|c|}
\hline \multicolumn{2}{|c|}{ DOCENTES } & \multirow[b]{2}{*}{ TDAES } & \multicolumn{2}{|c|}{ ESTUDIANTES } \\
\hline $\begin{array}{l}\text { IMPRESCINCRBLZ } \\
\text { E MMPOKTANTE }\end{array}$ & $\begin{array}{l}\text { POO } \\
\text { IMPORTANTE Y } \\
\text { CAREITE DE } \\
\text { DOORTANCIA }\end{array}$ & & $\begin{array}{l}\text { SIEMPREY } \\
\text { CON } \\
\text { FRECUENCA }\end{array}$ & $\begin{array}{c}\text { CAST } \\
\text { NUHCA } \\
\text { NUNCA }\end{array}$ \\
\hline$\%$ & $\%$ & - I. VALORES ETIOO-MORALES & $\%$ & $\%$ \\
\hline 9924 & 0.72 & 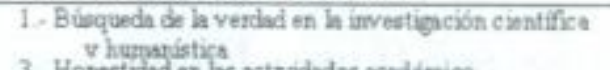 & 78.56 & 2144 \\
\hline 100.00 & 0.00 & $\begin{array}{l}\text { 3- Honestride en las actrvidades acadimico- } \\
\text { sdministrativas }\end{array}$ & 65.88 & 34.12 \\
\hline 92.81 & 3.19 & 12. Concistencia entre las declanciones y los actos & 59.63 & 40.47 \\
\hline \multirow[t]{2}{*}{7660} & 23.40 & 16. Autenticidad en las operones ideologicas avunidas & 59.48 & 4052 \\
\hline & & $\begin{array}{l}\text { If VALORESREIACIONADOSCONLA } \\
\text { DEMOCRACIA }\end{array}$ & & \\
\hline 100.00 & 0.00 & $\begin{array}{l}\text { 2. Respeto por h liberted de pensamuento } y \text { de } \\
\text { exoresión }\end{array}$ & 87.15 & 1285 \\
\hline 9929 & 0.71 & 4. Respeto a la petsona humana & 88.46 & 1154 \\
\hline 9383 & 6.17 & 7. Disporicíbe pan el diábgo & 77.50 & 2250 \\
\hline \multirow[t]{2}{*}{9433} & 5.67 & 8. Tolerancis en el divbogo & 76.06 & 23.84 \\
\hline & & - III VALORES PEDAGOGICOS & & \\
\hline 97.86 & 2.14 & 5- Actualización del proceso ensefianza - sprendiraje & 65.55 & 3445 \\
\hline 96.40 & 3.60 & 6.- Objetividad en la calificación del eprendiraje & 75.07 & 2493 \\
\hline 9424 & 5.76 & 9. Cotopromiso con la cabdad do heducteion & 67,38 & 32.22 \\
\hline \multirow[t]{2}{*}{95.68} & 432 & 13. Cumplinuento de is programado en el sylbubue. & 68.81 & 31.19 \\
\hline & & - IV. VALORES POLITICO-SOCIALES & & \\
\hline 92.91 & 7.09 & 10. Anilisis y seflexión sobre ha problemática del peis & 67.76 & 32.24 \\
\hline 9300 & 7.00 & 11. Compromieo con la recuperación moral del pais. & 5831 & 41.69 \\
\hline 92.03 & 797 & 14. Compromiso con el desanollo del pais & 60.73 & 39.27 \\
\hline 82.02 & 17,98 & $\begin{array}{l}\text { 15. Examen critico de las politicas gubemamentales } \\
\text { sobre los problemas sconotricos y exciales del peis. }\end{array}$ & 53.07 & 46.93 \\
\hline 84.40 & 1560 & $\begin{array}{l}\text { 19. Compromiso son la efirmsción de la identidad } \\
\text { nacional }\end{array}$ & 6633 & 33.67 \\
\hline \multirow[t]{2}{*}{82.64} & 16.46 & 20.- Recharo a los prejuicios étricos y regionales. & 6897 & 31.03 \\
\hline & • & $\begin{array}{l}\text { V. VALORES RELACIONADOS CON LA } \\
\text { PROYECCION SOCIAL }\end{array}$ & & \\
\hline 9038 & 9.42 & \multirow{2}{*}{$\begin{array}{l}\text { 17. Proyeceibn Social Universiteme hac ia la comunided. } \\
\text { 18. Soldaridad con be sectores poblacionales mis } \\
\text { desfervorecidos. }\end{array}$} & 5607 & 4393 \\
\hline 8832 & 11.68 & & 54,41 & 45.59 \\
\hline
\end{tabular}

Análogamente, el ítem 11: (Compromiso con la recuperación moral del país, del área Valores Político-Sociales), es altamente valorado por los docentes: 93\% (sumando 48.25 y 44.75, correspondientes a Imprescindible e Importante) mientras que, los estudiantes, en un porcentaje de $41.69 \%$ (sumando 36.06 y 5.63), manifiesta que tal valoración Casi nunca o Nunca se cumple en la realidad.

Algo semejante, aunque más acentuadamente, ocurre con los ítemes 14 y 15 (Compromiso con el desarrollo del país, y Examen crítico de las políticas gubernamentales sobre los problemas económicos y sociales del país) en los cuales la valoración de los docentes es alta: $92.03 \%$ (sumando 36.23 y 55.00 ) y $82.02 \%$ (sumando 23.02 y 59.00 ), mientras que los estudiantes declaran que Casi nunca o Nunca se cumplen tales valoraciones en la realidad: en el $39.27 \%$ (sumando 35.08 y 4.19 en el ítem 14) y en el $46.92 \%$ (sumando 39.74 y 7.18en el ítem 15).

Análogamente, pero de modo más acentuado aún, ocurre con los ítemes 17: Proyección Social Universitaria hacia la comunidad y 18: Solidaridad con los sectores 
poblacionales más desfavorecidos del área de Valores relacionados con la proyección social. En efecto, los docentes valoran en alto grado ambos contenidos: $90.58 \%$ en el ítem 17 (sumando 31.88 y 58.70) Y 88.32\% en el ítem 18 (sumando 29.93 y 58.39), mientras que los estudiantes declaran que Casi nunca o Nunca se cumplen tales valoraciones en la realidad: el $43.93 \%$ en el ítem 17 (sumando 38.81 y 5.12) Y el $45.59 \%$ en el ítem 18 (sumando 38.79 y 6.80 ).

El cuadro $\mathrm{N}^{\circ} 3$ tiene un contenido similar al cuadro $\mathrm{N}^{\circ} 2$, pero presenta los resultados en forma resumida: los valores porcentuales se consignan por Áreas de valores y no por Ítemes aislados. Confirmando lo hallado en el cuadro $\mathrm{N}^{\circ} 1$ se aprecia que las mayores disonancias entre la valoración de los profesores y la percepción de los estudiantes se encuentran en orden decreciente en las siguientes áreas:

V.- Valores relacionados con la proyección social. Alta valoración de los docentes: $89.45 \%$; mientras que el $44.79 \%$ de los estudiantes manifiestan que Casi nunca o Nunca se plasman tales valoraciones en la práctica docente.

IV.- Valores políticos - sociales. Alta valoración de los docentes: 87.82\%; mientras que el $37.45 \%$ de los estudiantes declaran que tales valoraciones no se aplican en la realidad.

I.- Valores ético - morales. Alta valoración de los docentes: 92.10\%; mientras que el $34.07 \%$ de los estudiantes manifiestan que tales valoraciones no se cumplen en la realidad.

III.- Valores pedagógicos. Alta valoración de los docentes: 91.05\%; mientras que el $34.07 \%$ de los estudiantes declaran que tales valoraciones no se plasman en la práctica docente.

Se advierte, además, que la disonancia es marcadamente inferior y, correlativamente, la consonancia o conformidad es mayor en el Área 11- Valores relacionados con la democracia. En efecto, hay una alta valoración de los docentes: 96.87\%; y una percepción altamente positiva por parte de los estudiantes: $82.32 \%$. Estos señalan que Siempre o Con frecuencia se cumplen tales valoraciones en la realidad. Sólo una pequeña proporción, esto es, el $17.68 \%$ de los estudiantes, manifiesta que dichas valoraciones no se plasman en la realidad.

El grado de significación de los porcentajes correspondientes a las respuestas de docentes y estudiantes se determinaron en base a su error estándar al nivel del 0.01. Lo referente a la dirección e intensidad de las respuestas, así como su relación de contingencia con el tiempo de permanencia de los estudiantes en la universidad, según carreras, se determinó mediante el estadístico CHP, al nivel de significación del 0.001. Los resultados de estos procesamientos estadísticos figuran en los cuadros numerados del tres al doce en el Informe Ampliado correspondiente al Proyecto de Investigación de código 021801025 del Instituto de Investigaciones Psicológicas de la Facultad de Psicología de la Universidad Nacional Mayor de San Marcos.

Al relacionar, mediante el estadístico CHP, en un cuadro de contingencia de doble entrada, por un lado, la dirección de cómo perciben los estudiantes de diversas carreras la aplicación que en la práctica docente tiene las valoraciones formuladas por los profesores y, por otro lado, el hecho de que los estudiantes estén cursando los primeros o los últimos años de estudios de sus respectivas planes curriculares, según áreas de valores, encontramos resultados heterogéneos. 
Como se vio anteriormente, las respuestas de los estudiantes presentan predominio de consonancias con las valoraciones de los docentes, aunque las disonancias, pese a ser cuantitativamente menores, poseen un significado importante por su índole.

El cruce de variables responde al interés de examinar las variaciones de las respuestas de los estudiantes en función del tiempo de su permanencia en la universidad, según áreas de valores. La hipótesis subyacente en esta operación sostiene que la percepción de los estudiantes acerca de la realidad académica en la que se desenvuelven sufre modificaciones, desde una percepción algo idealizada al comienzo hasta una percepción más realista en los últimos años, no exenta de cierto desencanto y de fuerte sentido crítico.

Entre los estudiantes de Psicología encontramos que sólo en las áreas 1. Valores ético morales y IV. Valores político - sociales se verifica, al nivel de significación del 0.001, el predominio de sus respuestas (Siempre y Con frecuencia) sobre (Casi nunca y Nunca), las mismas que son consonantes con las valoraciones de los docentes. Las respuestas estudiantiles se modifican a través del tiempo de su permanencia en la universidad, en el sentido de que en los últimos años disminuye el referido grado de consonancia entre las valoraciones de los docentes y la percepción de los estudiantes acerca de su aplicación en la praxis enseñanza - aprendizaje.

No descartamos que pueda haber en esto algún efecto de cohorte, esto es, características específicas de promociones, pero ello se minimiza como componente de la varianza total del fenómeno en estudio debido a que los estudiantes que integran la muestra pertenecen a diferentes promociones y, por otro lado, la duración de los estudios profesionales de las diferentes carreras no son iguales y, sin embargo, la tendencia en mención se mantiene.

A diferencia de lo anterior, en las áreas 11 Valores relacionados con la democracia, ID Valores pedagógicos y $\mathrm{V}$ Valores relacionados con la proyección social, la aludida consonancia no sufre mengua, lo cual es una interpretación del hecho de que en tales casos el estadístico CHP de independencia no llega a ser significativo ni al 0.05.

Los estudiantes de Psicología presentan una tendencia netamente definida. En todas las áreas, a mayor tiempo de permanencia en la universidad, mayor proporción de respuestas que afirman que Casi nunca o Nunca las valoraciones de los docentes se plasman en la realidad académica. En todas las áreas el porcentaje de las indicadas respuestas de los estudiantes de los últimos años es mayor que el de los estudiantes de los primeros años. Sin embargo sólo en las áreas 1. Valores ético - morales y IV Valores político- sociales las diferencias entre las respuestas de los estudiantes de los últimos años y de los primeros años son significativas al nivel del 0.001. En las demás áreas, tales diferencias no llegan a ser significativas al nivel del 0.05 .

En este contexto merecen destacarse los datos puntuales en el área de Valores ético morales. Los porcentajes de las respuestas que expresan disonancia presentan un marcado incremento, a saber: estudiantes de los primeros años: $34.1 \%$; estudiantes de los últimos años: $51.33 \%$. En el área de los valores político - sociales: estudiantes de los primeros años: $39.81 \%$; estudiantes de los últimos años: $53.33 \%$. Como se aprecia, las diferencias porcentuales son marcadas.

En Obstetricia y en Enfermería, no se presentan diferencias significativas entre las respuestas de las estudiantes de los primeros años y la de los últimos años, excepto en el área IV, valores políticos sociales, en la cual se registra una diferencia significativa al nivel del 0.001 , de sentido contrario a nuestra hipótesis de trabajo, esto es, la disonancia entre los valores proclamados por los profesores y la percepción de su aplicación en la praxis docente se presenta alta en los primeros años de estudio $(59.40 \%)$ y luego desciende a menos de la mitad (26.60 \%), lo cual significa, en lo esencial, que conforme pasa el tiempo las alumnas conocen mejor la mentalidad y la conducta de sus profesoras así como la realidad del contexto universitario y con ello van descubriendo y constatando la 
conformidad o consonancia entre las valoraciones de las profesoras y su aplicación en la praxis docente. En otras palabras, conforme los estudiantes avanzan en el cumplimiento de su plan curricular, perciben, cada vez más, que las valoraciones político-sociales de las profesoras se cumplen en la realidad académica.

Los estudiantes de Educación presentan también una tendencia claramente definida en relación con el tiempo de permanencia en la universidad, pero diametralmente opuesta a la de los estudiantes de Psicología. Mientras que en éstos a mayor tiempo en la universidad mayor es el grado de disonancia, en el caso de los estudiantes de Educación la relación que define la tendencia es inversa, a saber, a mayor tiempo en la universidad menor es el grado de disonancia entre las valoraciones formuladas por los profesores y las percepciones estudiantiles de su aplicación en la praxis docente.

En efecto, en todas las áreas el porcentaje de los estudiantes de los últimos años que manifiestan que casi nunca o nunca se plasman en la realidad las valoraciones formuladas por los docentes son invariablemente menores que los porcentajes de los estudiantes de los primeros años.

Las diferencias son significativas al nivel del 0.001 en las áreas 1. Valores ético morales; II. Valores relacionados con la democracia; y III. Valores pedagógicos. Sin embargo, en las áreas IV. Valores político sociales y V. Valores relacionados con la proyección social, las diferencias no llegan a ser significativas.

Es de suponerse, que conforme se afirma el proceso de interacción entre estudiantes y docentes, ambas partes llegan a conocerse mejor y se produce una mayor compenetración con el contexto universitario. Ello contribuiría a que las percepciones de los estudiantes tengan progresivamente mayor fundamento al mantenerse, incrementarse o disminuir las consonancias y disonancias. 


\section{RESULTADOS A NIVEL DE ÍTEMES}

Cuadro $N^{\circ}$ 13: Principales disonancias a nivel de ítemes, en orden decreciente, entre las valoraciones formuladas por los profesores y la percepción de los estudiantes acerca de la aplicación de tales valoraciones en la praxis docente.

\section{ITEM}

15. Examen crítico de las políticas gubernamentales sobre los problemas económicos y sociales del país.

18. Solidaridad con los sectores poblacionales más desfavorecidos.

17. Proyección social universitaria hacia la comunidad.

11. Compromiso con la recuperación moral del país.

16. Autenticidad en las opciones ideológicas asumidas.

12. Consistencia entre las declaraciones y los actos.

14. Compromiso con el desarrollo del país.

5. Actualización del proceso enseñanza aprendizaje.

3. Honestidad en las actividades académico-administrativas.

19. Compromiso con la afirmación de la identidad nacional

10. Análisis y reflexión sobre la problemática del país

9. Compromiso con la calidad de la educación

13. Cumplimiento con 10 programado en el syllabus

20. Rechazo a los prejuicios étnicos y regionales
$46.92 \%$

$45.59 \%$

$43.93 \%$

$41.69 \%$

$40.52 \%$

$40.37 \%$

$39.27 \%$

$34.45 \%$

$34.12 \%$

$33.67 \%$

$32.23 \%$

$32.22 \%$

$31.19 \%$

$31.03 \%$

A nivel de ítemes individualmente considerados las respuestas de los estudiantes, dispuestas en orden decreciente, según la magnitud de los porcentajes, se presentan en el cuadro $\mathrm{N}^{\circ} 13$.

A fin de mostrar con claridad las respuestas más destacadas de los estudiantes a los ítemes individualmente considerados hemos clasificado a éstos en tres categorías, a saber:

a) Los ítemes en los que los estudiantes contestan en un $40 \%$ o más que «Casi Nunca o Nunca» perciben que las valoraciones de los docentes se plasman en la realidad.

b) Los ítemes en los cuales el citado porcentaje es mayor que 30 y menor que 40

c) Los ítemes en los cuales el referido porcentaje es menor marcadamente bajo y, por lo tanto, el nivel de consonancia es alto.

Los datos son bastantes elocuentes por si mismos. En cada caso consignamos, a manera de indicador, entre paréntesis, el porcentaje de estudiantes que manifiestan que casi nunca o nunca perciben que las valoraciones de los profesores sobre los temas aludidos por los respectivos ítemes se plasman en la actividad docente.

a) Primer grupo. Los ítemes del primer grupo revelan, a través de las percepciones de lo estudiantes, que un apreciable porcentaje de docentes no suele examinar críticamente en el plano académico obviamente- las políticas gubernamentales sobre los problemas económicos y sociales del país (46.92\%); revelan también, en cierto grado, actitudes de ausencia de solidaridad con los sectores poblacionales más desfavorecidos (45.59\%) Y poco interés en la proyección social universitaria (43.93\%). Del mismo modo, se detecta que una considerable proporción de docentes no manifiesta en los hechos 
compromiso con la recuperación moral del país (41.69 \%) -problema central en los tiempos que corren y de propiedades corrosivas respecto al presente y al futuro de la nación peruana- . Los porcentajes de respuesta que comentamos ponen también de relieve que un apreciable porcentaje de docentes no pone en práctica los valores éticosmorales que proclama tales como la «autenticidad en las opciones ideológicas asumidas» $(40.52 \%)$ y la «consistencia entre las declaraciones y los actos» $(40.37 \%)$.

b) Segundo grupo. Pese a que las disonancias incluidas en el segundo grupo no son las más categóricas, algunas de ellas, por su especial significado, llaman a reflexión y generan inevitablemente preocupación. Tal es el caso de que una cierta proporción de docentes universitarios (39.27\%), no manifieste en los hechos «compromiso con el desarrollo del país», ni con «el análisis y reflexión sobre la problemática del país» $(32.23 \%)$, ni con «el rechazo a los prejuicios étnicos y regionales» $(31.03 \%)$.

Preocupa igualmente que, en el campo pedagógico, ocurra algo semejante respecto a «la actualización del proceso enseñanza aprendizaje» $(24,45 \%)$ y el «compromiso con la calidad de educación» $(32.22 \%)$.

Finalmente, no es precisamente reconfortante que acontezca algo similar en cuanto a «honestidad en las actividades académico administrativas» (34.12\%).

c) Tercer grupo. Las consonancias que caracterizan al tercer grupo entrañan un significado positivo sumamente importante. Su sentido es concordante con las tradiciones conspicuas de San Marcos. Nos referimos a los temas relacionados con la democracia. En efecto, los ni veles de disonancia son marcadamente bajos (17.68\%, en promedio), lo cual implica que la gran mayoría de los estudiantes perciben que los docentes proclaman y practican en los hechos valores tan importantes para la vida civilizada, la democracia y el desarrollo integral del país como, «el respeto a la persona humana» $(11,54 \%)$, « respeto por la libertad de pensamiento y de expresión « $(12,85$ $\%)$, « disposición para el diálogo» $(22.50 \%)$ y « tolerancia en el diálogo « $(23.84 \%)$. Por otro lado, «la búsqueda de la verdad en la investigación científica y humanística» (21.44\%) es un valor ético- moral alrededor del cual hay importante consonancia.

\section{DISCUSIÓN DE LOS RESULTADOS}

Una limitación del estudio realizado que debe tenerse en cuenta en la interpretación de los resultados reside en que la muestra de docentes y estudiantes no es aleatoriamente representativa de las numerosas carreras que ofrece San Marcos ni tiene el tamaño técnicamente deseable; es, más bien, una muestra intencional y, en consecuencia, sus conclusiones no son categóricas, sino son susceptibles de confirmarse o modificarse a través de otros estudios que superen las limitaciones señaladas.

Al contrastar las valoraciones que los profesores formulan sobre un conjunto de actividades y aspectos sustanciales de la universidad con la percepción que tienen los estudiantes de la aplicación de tales valoraciones en la praxis docente se encuentra que la consonancia o conformidad entre ambos términos del contraste es mayoritario. Sin embargo, el hecho de que tal consonancia no sea total, como teóricamente se espera para efectos del contraste estadístico, sino que conlleve disonancias o disconformidades en un porcentaje apreciable de casos, patentiza la existencia de un problema ético-social, en base a lo cual rechazamos nuestra hipótesis nula de ausencia de disonancias a un nivel alto de significación( 0.001 al aplicar el estadístico CHI cuadrado, salvo unos pocos casos en los que la significatividad fue del 0.01) y aceptamos la correspondiente hipótesis alternativa que afirma la existencia de tales disonancias.

Al comparar las respuestas de los estudiantes de diferentes carreras entre sí, independientemente del tiempo de permanencia del tiempo de los estudiantes en la universidad, 
no se encuentran variaciones significativas en los niveles de consonancia- disonancia.

Las variaciones de las respuestas de los estudiantes en relación con el tiempo de su permanencia en la universidad son marcadas y heterogéneas. Así, los estudiantes de Psicología y de Educación presentan tendencias definidas, pero diametralmente contrapuestas. En tanto que en Psicología la proporción de respuestas que implican disonancia (casi nunca y nunca), en todas las áreas de valores, se incrementa en los estudiantes de los últimos años, en Educación disminuye notablemente.

Entre las estudiantes de Obstetricia y Enfermería no encontramos tendencia alguna, pero adquiere relieve el hecho que en el área IV. Valores político - sociales, de modo similar a lo que ocurre en Educación, las respuestas de los estudiantes de los últimos años que implican disonancia se reducen marcadamente.

Aparte de la suposición plausible de que tales variaciones puedan deberse parcialmente al mejor conocimiento recíproco entre alumnos y docentes, producto de la interacción propia de la vida universitaria, no tenemos ningún otro elemento de juicio que nos permita conjeturar siquiera sobre las causas de las direcciones específicas que adoptan dichas variaciones

Como se expuso anteriormente, el análisis de las respuestas de los estudiantes, a nivel de ítemes individualmente considerados, pone de relieve, en un ordenamiento que empieza por lo positivo, una notable consonancia entre las valoraciones que formulan los profesores y la percepción de los estudiantes sobre su aplicación en la praxis docente en lo que concierne a los valores relacionados con la democracia (respeto a la persona humana, cultivo del diálogo) y a los valores ético morales ( búsqueda de la verdad en la investigación científica y humanística).

Tales hallazgos son congruentes con el nivel académico de San Marcos y con su conspicua tradición de simbolizar la conciencia moral y crítica del país.

Por otro lado, se destaca una apreciable disonancia, que entraña falta de examen de las políticas gubernamentales sobre los problemas económicos y sociales del país, débil solidaridad con los sectores poblacionales más desfavorecidos, poco interés en la proyección social universitaria hacia la comunidad, falta de compromiso con la recuperación moral del país. Asimismo, se encuentra que una cierta proporción de docentes no pone en práctica los valores ético-morales que proclama tales como la autenticidad en las opciones ideológicas asumidas y la consistencia entre las declaraciones y los actos.

Estos hechos son contrarios a las tradiciones de San Marcos, especialmente aquellas que denotan cierto grado de insensibilidad social, así como indiferencia respecto a la corrupción que asola a la sociedad peruana y cierto desinterés por los valores pedagógicos.

Se desprende de tales constataciones la necesidad de afirmar las fortalezas y promover una toma de conciencia colectiva sobre los problemas ético-sociales expuestos, entre las autoridades, docentes y estudiantes y luego,a partir de ello, concebir, diseñar y ejecutar acciones diversas para revertir tal situación.

Como colofón de estos comentarios y en orden a situarlos en su real dimensión, conviene tener en cuenta algunas consideraciones que los relativizan. En esta perspectiva, puntualizamos que, en general, las consonancias son marcadamente mayores que las disonancias, pero, por otro lado, las disonancias, siendo menores que aquellas, son significativas debido a que las expectativas sociales acerca del rol docente en la universidad son, por definición, altas, tanto más tratándose de San Marcos por su alto nivel académico y su tradición como conciencia moral y crítica del país. En este contexto es que impacta negativamente, por ejemplo, que el $30 \%$ de los estudiantes perciban que Casi Nunca o Nunca se plasman en los hechos las valoraciones verbales que formulan los profesores respecto al «compromiso con el desarrollo del país» o en torno al «compromiso con la recuperación moral del país» y, al propio tiempo, impactan positivamente, por ejemplo, que 
la disonancia sea mínima y, consecuentemente, la consonancia sea alta en lo que atañe a los valores ligados a la democracia, por ejemplo, los relativos al respeto a la persona humana, al cultivo del diálogo y a los valores de sentido ético - moral como «búsqueda de la verdad en la investigación científica y humanística».

\section{CONCLUSIONES}

] Dada las limitaciones muestrales las conclusiones de la presente investigación sólo tienen carácter aproximativo

2. Las consonancias entre las valoraciones que formulan los profesores sanmarquinos sobre diversos aspectos y actividades propias de la institución universitaria y la percepción que tienen los estudiantes, en general, acerca de la aplicación de tales valoraciones en la praxis docente es cuantitativamente mayor que las disonancias.

Sin embargo, estas últimas, pese a fluctuar sólo entre el 30 y el $47 \%$ de las respuestas de los estudiantes, son significativas por cuanto revelan una problemática que concierne a aspectos sustanciales de la formación de profesionales, investigadores y futuros dirigentes del país

3. Las mayores disonancias entre las valoraciones formuladas por los docentes y la percepción de los estudiantes, en orden decreciente, se resumen en el siguiente cuadro: 


\begin{tabular}{|c|c|c|}
\hline $\begin{array}{l}\text { DOCENTES } \\
\text { Imprescindible } \\
\text { Importante }\end{array}$ & ÁREAS & $\begin{array}{l}\text { ESTUDIANTES } \\
\text { Casi nunca, nunca }\end{array}$ \\
\hline 92.10 & $\begin{array}{l}\text { II. Valores relacionados con la democracia } \\
\text { 2. Respeto por la libertad de pensamiento y } \\
\text { expresión } \\
\text { 4. Respeto a la persona humana } \\
\text { 7. Disposición para el dialogo. } \\
\text { 8. Tolerancia en el dialogo. } \\
\text { V. Valores relacionados con la Proyección } \\
\text { Social } \\
\text { 17. Proyección social universitaria hacia la } \\
\text { comunidad } \\
\text { 18.Solidaridad con los sectores poblacionales } \\
\text { más desfavorecidos } \\
\text { IV. Valores político-sociales } \\
\text { 10. Análisis y reflexión sobre la problemática } \\
\text { del país. } \\
\text { 11. Compromiso en la recuperación moral del } \\
\text { país } \\
\text { 14. Compromiso con el desarrollo del país } \\
\text { 15. Examen crítico de las políticas } \\
\text { gubernamentales sobre los problemas } \\
\text { económicos y sociales del país } \\
\text { 19. Compromiso con la afirmación de la } \\
\text { identidad del país } \\
\text { 20. Rechazo a los prejuicios étnicos y } \\
\text { regionales. } \\
\text { I. Valores ético-morales } \\
\text { 1. Búsqueda de la verdad en la investigación } \\
\text { científica y humanística } \\
\text { 3. Honestidad en las actividades académico- } \\
\text { administrativas } \\
\text { 12.Consistencia entre las declaraciones y los } \\
\text { actos } \\
\text { 16. Autenticidad en las opciones ideológicas } \\
\text { asumidas } \\
\text { III. Valores pedagógicos. } \\
\text { 5. Actualización del proceso enseñanza } \\
\text { aprendizaje } \\
\text { 6. Objetividad en la calificación del aprendizaje. } \\
\text { 9. Compromiso en la calidad de docencia } \\
\text { 13. Cumplimiento del lo programado en el } \\
\text { syllabus }\end{array}$ & 34.07 \\
\hline
\end{tabular}

4. No se encuentran diferencias significativas de los niveles de consonancia - disonancia entre estudiantes agrupados según las carreras que siguen.

5. Al comparar las respuestas de disonancia de estudiantes de los primeros años del plan curricular con los de los últimos años, según carreras y según áreas de valores, se constata que los estudiantes de Psicología y los de Educación presentan tendencias definidas.. pero diametralmente contrapuestas. Mientras que en Psicología los niveles de disonancia son mayores en los estudiantes de los últimos años, lo cual implica que la percepción positiva inicial se va deteriorando progresivamente, en Educación ocurre lo 
contrario: los niveles de disonancia son menores en los estudiantes de los últimos años, hecho que denota un progresivo incremento de las consonancias entre las estudiantes de Obstetricia y de Enfermería se encuentra que sólo en el área de valores político sociales, de modo similar a lo que ocurre en Educación, los niveles de disonancia son menores en los estudiantes de los últimos años, con el significado antes anotado.

6. El análisis de los resultados a nivel de ítemes individualmente considerados, mediante el procedimiento de clasificarlos en tres categorías según la magnitud de las disonancias que conllevan, pone de relieve lo siguiente:

a. ítemes en cuyas respuestas los estudiantes manifiestan en un cuarenta por ciento o más que casi nunca o nunca perciben la aplicación de las valoraciones de los profesores en la praxis docente; $y$

b. ítemes en cuyas respuestas los estudiantes manifiestan en un porcentaje mayor que $30 \mathrm{y}$ menor que 40 por ciento ocurre la disonancia antes referida

c. ítemes en los que la disonancia es marcadamente baja y consecuentemente la consonancia es baja.

\section{ITEM}

\section{CASI NUNCA O NUNCA}

15. Examen crítico de las políticas gubernamentales

$46.92 \%$

16. sobre los problemas económicos y sociales del país.

$45.59 \%$

18. Solidaridad con los sectores poblacionales más $43.9 \%$ desfavorecidos.

17. Proyección social universitaria hacia la comunidad. $41.69 \%$

11. Compromiso con la recuperación moral del país. $40.52 \%$

16. Autenticidad en las opciones ideológicas asumidas. $40.37 \%$

12. Consistencia entre las declaraciones y los actos.

14. Compromiso con el desarrollo del país. $39.7 \%$

5. Actualización del proceso enseñanza aprendizaje. $34.45 \%$

3. Honestidad en las actividades académico administrativas. $34.12 \%$

19. Compromiso con la afirmación de la identidad nacional $33.67 \%$

10. Análisis y reflexión sobre la problemática del país $\quad 32.23 \%$

9. Compromiso con la calidad de la educación $32.22 \%$

13. Cumplimiento con10 programado en el syllabus $31.19 \%$

20. Rechazo a los prejuicios étnicos y regionales $31.03 \%$

Los ítemes incluidos en el tercer grupo (c) poseen un significado positivo muy importante. Su sentido es concordante con las tradiciones conspicuas de San Marcos. Nos referimos a los valores relacionados con la democracia y algunos de los valores ético - morales tales como: «el respeto a la persona humana» $(11,54 \%)$, «respeto por la libertad de pensamiento y de expresión» $(12,85 \%)$, «disposición para el diálogo» $(22.50 \%)$ y « tolerancia en el diálogo « $(23.84 \%)$. Por otro lado, «la búsqueda de la verdad en la investigación científica y humanística» $(21.44 \%)$ es un valor ético- moral alrededor del cual hay importante consonancia.

7. Los resultados del procesamiento estadístico de la información en torno al problema central de la investigación mediante el uso del estadístico $\mathrm{CHI}^{2}$, nos permiten desechar la hipótesis nula (Ho) al nivel de 0.001 de significación y, consecuentemente, quedamos con la hipótesis alternativa $(\mathrm{Hi})$, según la cual existe un problema ético de disonancia 
entre las valoraciones formuladas por los profesores y la percepción de los estudiantes de su aplicación en la praxis docente

\section{RECOMENDACIONES}

1. Afirmar las fortalezas de la comunidad universitaria sanmarquina en torno a los valores relacionados con la democracia que presentan alto grado de consonancia.

2. Promover en la comunidad universitaria la toma de conciencia de los problemas éticos identificados en el presente estudio, a través de la realización de conversatorios, foros, publicaciones, etc. , a fin de motivar a profesores, estudiantes y autoridades a iniciar acciones permanentes de reflexión e interacción grupal orientadas a la superación gradual de los aludidos problemas.

\section{REFERENCIAS BIBLIOGRÁFICAS}

Camps, V. (ed.) Concepciones de la ética. Madrid. Trotta. 1992

Cortina, A. Y Martínez, E. (1998). Ética. Madrid: Akal.

Etxeberria, X. (1995) Ética básica. Bilbao: Universidad de Deusto.

Gonzáles, L. J. (1996). Ética. Santafe de Bogotá: El Búho.

Guariglia, O. Cuestiones morales. Madrid. Trotta. 1996

Polo S, Miguel A.( 1996). Ética y crisis moral. Lima, Perú: Textos.

Singer, P. Compendio de ética. La Educación superior en el Siglo XXI, Tomo II, UNESCO, 1997.

Cruz Ramírez, J. (1996). México: Editorial Iberoamericana.

Xirau, J. Carácter básico y universal del humanismo con valores, su manifestación en el pensamiento humanista cubano.

Quintana, C. J. (1998). Pedagogía y axiología, la educación ante los valores. Madrid

Odero, J. M. Ética de los valores y coherencia existencial. Pamplona: Universidad de Navarra. 